\title{
Helmintos gastrointestinales en aves acuáticas de la subcuenca alta del río Lerma, México
}

\section{Gastrointestinal helminth in waterfowl of the upper Lerma river sub-basin, Mexico}

\author{
Marcela Martínez-Haro ${ }^{\circledR}{ }^{\circledR}$ Petra Sánchez-Nava1, Guillermo Salgado-Maldonado² y Felipe de Jesús \\ Rodríguez-Romero ${ }^{1}$ \\ ${ }^{1}$ Centro de Investigación en Recursos Bióticos, Facultad de Ciencias, campus El Cerrillo, Universidad Autónoma del Estado de México. Carretera \\ Toluca-Atlacomulco Km. 14.5, 50200 Toluca, México. \\ ${ }^{2}$ Instituto de Biología, Universidad Nacional Autónoma de México. Apartado postal 70-153, 04510 México D. F. \\ \arbolblue@yahoo.com.mx
}

\begin{abstract}
Resumen. Se realizó un inventario y se calcularon los parámetros de infección de los helmintos gastrointestinales de 36 ejemplares de aves acuáticas pertenecientes a las familias Anatidae, Rallidae y Threskiornithidae, procedentes de la subcuenca alta del río Lerma, Estado de México, identificándose 20 especies: 9 tremátodos, 8 céstodos, 2 nemátodos y 1 acantocéfalo. De las 8 especies de céstodos, 6 son registros nuevos para el país y Pseudocorynosoma constrictum se registra por primera vez en Anas crecca, Anas discors, Oxyura jamaicensis y Fulica americana. Los helmintos que presentaron las prevalencias más altas fueron los céstodos Hymenolepis megalops y Sobolevicanthus krabbeella en Anas acuta, Anas clypeata, Anas cyanoptera y Anas crecca.
\end{abstract}

Palabras clave: aves acuáticas, Cestoda, Digenea, Nematoda, Acantocephala, prevalencia, abundancia.

\begin{abstract}
A survey of helminth parasites in 36 waterfowl species from the upper Lerma River, in central Mexico was conducted. A total of 20 helminth species were recorded, including 9 trematodes, 8 cestodes, 2 nematodes and 1 acanthocephalan. Six of the cestode species are recorded for the fist time from Mexican birds; the acanthocephalan Pseudocorynosoma constrictum is reported for the first time in Anas crecca, A. discors, Oxyura jamaicensis and Fulica americana. The highest prevalences were recorded for the cestodes Hymenolepis megalops and Sobolevicanthus krabbeella in Anas acuta, A. clypeata, A. cyanoptera and A. crecca.
\end{abstract}

Key words: aquatic birds, Cestoda, Digenea, Nematoda, Acantocephala, prevalence, abundance.

\section{Introducción}

Los humedales que conforman la subcuenca alta del río Lerma proporcionan alimento, refugio y un hábitat adecuado para un considerable número de especies de flora y fauna silvestre, algunas de las cuales son endémicas y se encuentran dentro de alguna categoría de riesgo (RAMSAR, 2010). Asimismo, estos sitios son apropiados para albergar tanto aves residentes como migratorias; entre otras, los miembros de las familias Anatidae, Rallidae y Threskiornithidae. En general, se tienen pocos datos sobre los helmintos que parasitan las aves en México; los digéneos son el grupo mejor documentado (Peréz-Ponce de León et al., 2007), seguidos por los acantocéfalos (García-Prieto et al., 2010); para otros helmintos, como céstodos y nemátodos, existen inventarios helmintológicos más puntuales (Farías y Canaris, 1986; Canaris y Lei-Ching, 1989; Scholz et al., 2002; Ortega-

Recibido: 20 noviembre 2010 ; aceptado: 07 junio 2011
Olivares et al., 2008; Gladden y Canaris, 2009). El objetivo principal del presente estudio fue realizar un inventario de los helmintos gastrointestinales de algunas aves acuáticas de la subcuenca alta del río Lerma en el Estado de México y calcular sus parámetros de infección.

\section{Materiales y métodos}

Las aves acuáticas fueron obtenidas por donación de cazadores en la zona, durante las temporadas cinegéticas de noviembre 2008-febrero 2009, junio-julio 2009 y noviembre 2009-febrero 2010, en 4 localidades a lo largo de la subcuenca alta del río Lerma (Fig. 1): laguna de Almoloya (Alm, 19¹1'20" N; 99²9’30" O), ciénega de Lerma (Cie, $19^{\circ} 14^{\prime} 31.0^{\prime \prime} \mathrm{N}$; 99'28'53.9” O), laguna de Atarasquillo (Atar, 19¹8'34.0” N; 99 29'50.8” O) y estanque El Cerri-

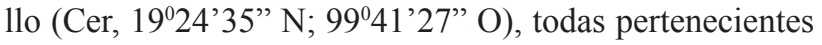
al Estado de México (Fig. 1).

Las aves se determinaron taxonómicamente con la ayuda de la guía de campo de Howell y Webb (1995). 


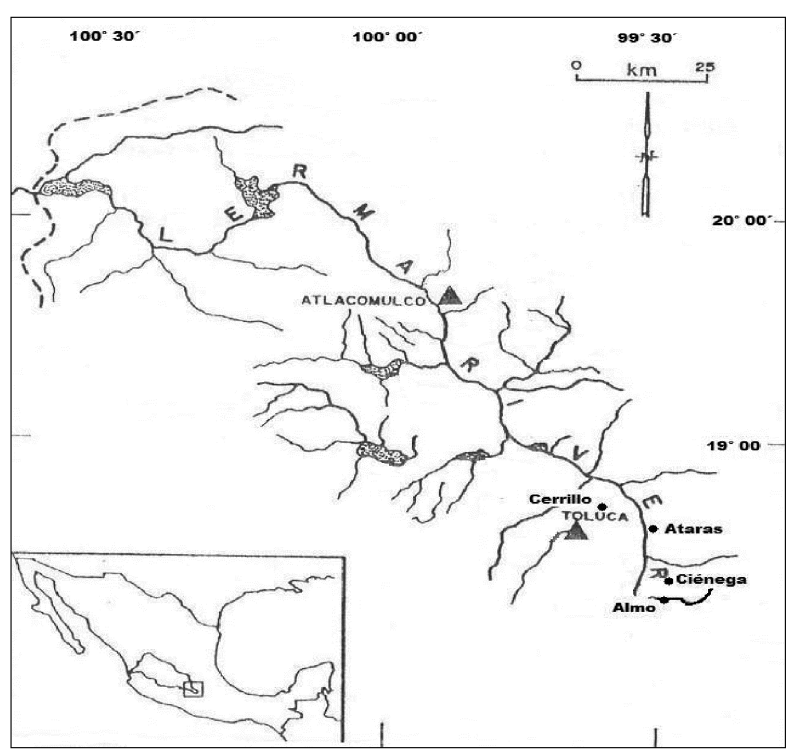

Figura 1. Subcuenca alta del río Lerma. Distribución de las aves hospederas en el área de estudio (modificado de Sanchez- Nava, 2004).

Las necropsias se hicieron en las 2 horas posteriores a la captura de los hospederos; en los casos en que no pudo examinarse inmediatamente el tubo digestivo, éste se fijó con formol al $4 \%$ caliente y se examinó posteriormente.

Todos los helmintos fueron extraídos del aparato gastrointestinal y contados in situ; tremátodos, acantocéfalos $\mathrm{y}$ céstodos se fijaron en formol al $4 \%$ caliente, mientras que los nemátodos en alcohol salino al $4 \%$ caliente; finalmente, todos los gusanos se conservaron en alcohol $70 \%$. Platelmintos y acantocéfalos se tiñeron con paracarmín de Meyer o hematoxilina de Delafield y se montaron en preparaciones permanentes en bálsamo de Canadá; los nemátodos se aclararon y montaron temporalmente con mezclas de glicerina y agua destilada en concentraciones de 1:20, 1:15. 1:10, 1:5, 1:1 (Salgado-Maldonado, 1979). Algunos escólices se fijaron con una mezcla de glicerina-picrato de amonio para estudiar los ganchos del rostelo (Scholz et al., 2002; Ortega-Olivares et al., 2008). La identificación de los helmintos se realizó utilizando las claves de Gibson et al. (2002), Jones et al. (2005), Czaplinzki y Vaucher (1994), Jones (1994), McDonald (1988) y Anderson (1986). Los parámetros de infección, prevalencia y abundancia se calcularon de acuerdo con los criterios de Margolis et al. (1982). Examinamos la similitud entre la composición de las comunidades de helmintos de las distintas especies de hospederos mediante el cálculo del índice de Sorensen (Krebs, 1999). Los ejemplares se depositaron en la Colección Nacional de Helmintos (CNHE 7548-7557 y CNHE 8056-8061),
Instituto de Biología, Universidad Nacional Autónoma de México.

\section{Resultados}

Se examinaron 36 aves acuáticas de las especies Anas acuta Linneo, 1758, A. clypeata L., 1758, A. crecca L., 1758, A. cyanoptera Vieillot, 1816, A. diazi Ridgway, 1886, A. discors L., 1766, Oxyura jamaicensis (Gmelin, 1789) (Anatidae), Fulica americana Gmelin, 1789 (Rallidae) y Plegadis chihi (Vieillot, 1817) (Threskiornithidae). En dichas aves, se determinó la presencia de 20 especies de helmintos: 9 tremátodos, 8 céstodos, 2 nemátodos y un acantocéfalo (Cuadro 1). Los céstodos representan $56.7 \%$ de los helmintos recuperados del total de aves examinadas, seguidos por los tremátodos con $36.5 \%$, y finalmente los nemátodos y acantócefalos con $2.1 \%$ y $4.5 \%$, respectivamente.

El céstodo Hymenolepis megalops registró la prevalencia más alta en $A$. clypeata y A. cyanoptera; sin embargo, su abundancia no se encontró entre las más significativas; asimismo, Sobolevicanthus krabbeella también se registró con prevalencia elevada en $A$. crecca pero su abundancia fue alta únicamente en $O$. jamaicensis. Las especies Diorchis americana en A. clypeata, Microsomacanthus sp. 1 en A. diazi y Cotylurus cornutus en A. cyanoptera se presentaron con prevalencias bajas, pero con abundancias altas (Cuadro 1).

La mayor riqueza de especies de helmintos se presentó en $A$. cyanoptera $(\mathrm{S}=8)$, A. acuta $(\mathrm{S}=7)$ y $A$. discors $(\mathrm{S}=$ 7). El análisis de similitud aplicando el índice de Sorensen, permite distinguir 3 grupos de hospederos, uno conformado por todos los de la familia Anatidae y otro por los de la Rallidae; $P$. chihi se separó en un grupo totalmente diferente a los 2 antes mencionados (Fig. 2).

\section{Discusión}

Se registraron en total 20 especies de helmintos en 9 especies de aves de los humedales de la subcuenca alta del río Lerma, en el Estado de México. Los grupos taxonómicos más frecuentes y abundantes en estos hospederos fueron los tremátodos y los céstodos, representados por especies que son comunes en varias especies de aves acuáticas en Norteamérica. Sin embargo, algunas especies de helmintos se registran por primera vez para México y en algunos casos se señalan nuevos registros de hospederos.

El grupo taxonómico con mayor número de especies en este estudio fue el de los tremátodos, lo que difiere de los datos referidos por Farias y Canaris (1986), Canaris y Lei-Ching (1989) y Gladden y Canaris (2009) quienes registraron menor número de digéneos en muestreos de 


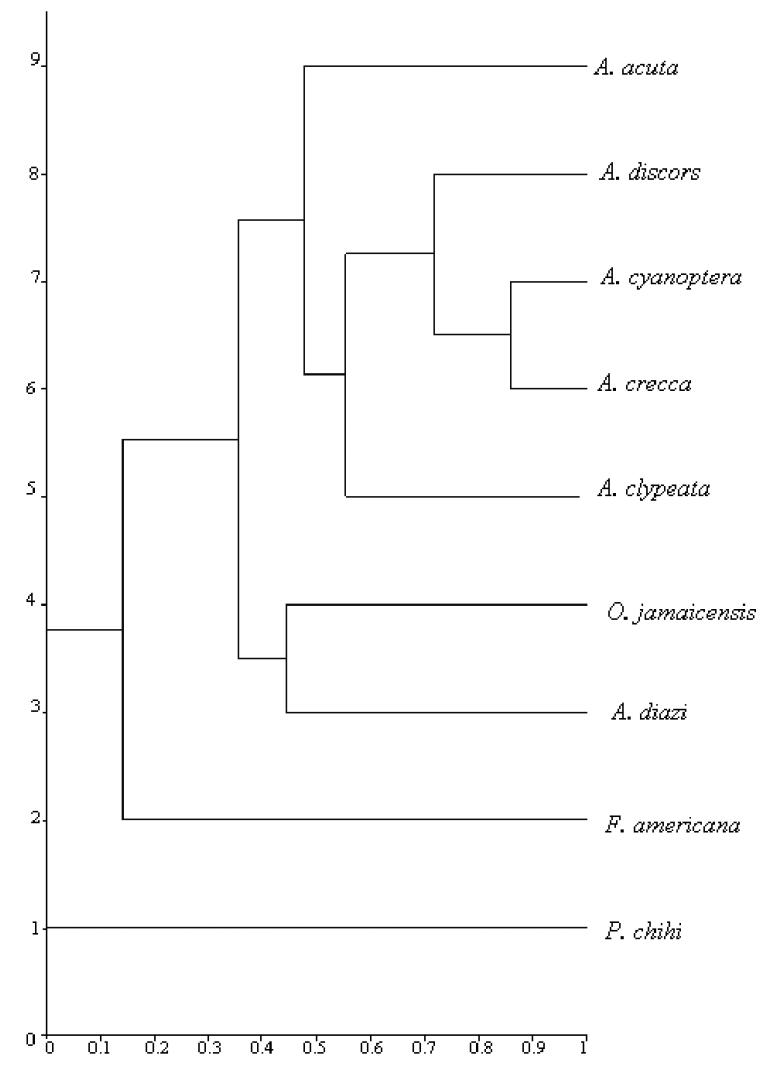

Figura 2. Cluster del índice de Sorensen, que agrupa a los hospederos de acuerdo con la composición taxonómica de las comunidades de helmintos gastrointestinales.

anátidos en el norte de México. Mas aún, consideramos que el número de especies de tremátodos registrados en esta investigación puede aumentar al resolver los problemas que plantea la diferenciación de las distintas especies de Notocotylus que aún estamos estudiando.

El presente estudio registra por primera vez para México los digéneos Echinoparyphium recurvatum en A. acuta, A. crecca, A. cyanoptera y A. discors; Echinostoma revolutum y Australapatemon minor en A. discors; el estrigéido Cotylurus brevis en A. acuta, A. crecca, $A$. cyanoptera y $O$. jamaicensis, así como $C$. cornutus en $A$. cyanoptera.

Los céstodos de la familia Hymenolepididae, parásitos comunes del grupo de las aves, no habían sido estudiados en México, probablemente debido a la complejidad de su manejo, ya que muchos de ellos son pequeños y delicados, por lo cual se fragmentan fácilmente impidiendo su adecuada determinación taxonómica (Czaplinski y Vaucher, 1994). En este estudio se identificaron 7 especies de la familia Hymenolepididae, 6 de las cuales (S. krabbeella, D. americana, Diorchis sp., Microsomacanthus sp. 1, Microsomacanthus sp. 2 y Fimbriaria fasciolaris) representan registros nuevos para el país. La séptima especie, H. megalops se considera generalista y con amplia distribución geográfica; los Anseriformes constituyen los hospederos preferenciales de esta especie, en Galliformes, Gruiformes y Ciconiformes se ha registrado en pocas ocasiones (Digiani, 2000); algunos estudios previos señalan prevalencias altas para este céstodo en América del Norte (Broderson et al., 1977; Wilkinson et al., 1977; Shaw y Kocan, 1980; Canaris et al., 1981); sin embargo, investigaciones realizadas en el norte del país registran prevalencias bajas a medias (Farias y Canaris, 1986; Canaris y Lei-Ching, 1989; Gladden y Canaris 2009), mientras que en el presente estudio se registran prevalencias altas, como en otros sitios de Norteamérica. Las especies $A$. crecca, $A$. discors, $O$. jamaicensis y $F$. americana son nuevos registros de hospedero para Pseudocorynosoma constrictum.

El análisis de similitud de la composición de las comunidades de helmintos entre las especies de hospederos examinadas permitió distinguir 3 grupos. La dieta, la conducta y la vagilidad de los hospederos son factores que influyen sobre la estructura de las comunidades de los helmintos (Fedynich et al., 1996; Poulin, 2007). La dieta de los patos se basa principalmente en vegetales y tubérculos (Colón-Quezada, 2009); sin embargo, $O$. jamaicensis es una especie buceadora, en tanto que $A$. diazi es filtrador y de talla grande, conductas que explican su separación en un subgrupo distinto al de los otros anátidos, ya que adquieren su alimento a mayor profundidad. Por su parte, A. crecca, A. cyanoptera y A. discors son aves pequeñas, no buceadoras, que se alimentan a poca profundidad, razón por la cual presentan mayor semejanza en la composición de sus comunidades de helmintos. Haukos y Neaville (2003) enfatizan que los patos de superficie, comparados con los patos buceadores, son más propensos a altas infecciones por $H$. megalops debido a su dieta generalista, lo que explica su presencia en éstos y su ausencia en $O$. jamaicensis.

Fedynich y Pence (1994) señalaron que la comunidad de helmintos se ve influenciada por su distribución estacional y abundancia en los hospederos migratorios, cuya vagilidad facilita el intercambio de los parásitos; sin embargo, Gladden y Canaris (2009) sugieren que los helmintos se pierden durante la ruta migratoria y su reemplazo se lleva a cabo en las áreas de invierno. En la zona de estudio que abarca este trabajo, Barragán-Sáenz et al. (2009) registraron cercarias de Notocotylus sp., y metacercarias de C. cornutus, E. revolutum y E. recurvatum en caracoles de agua dulce, mientras que Sánchez-Nava et al. (2004) registraron metacéstodos de Cyclustera ralli en Girardinichthys multiradiatus. El hallazgo previo de estas fases 


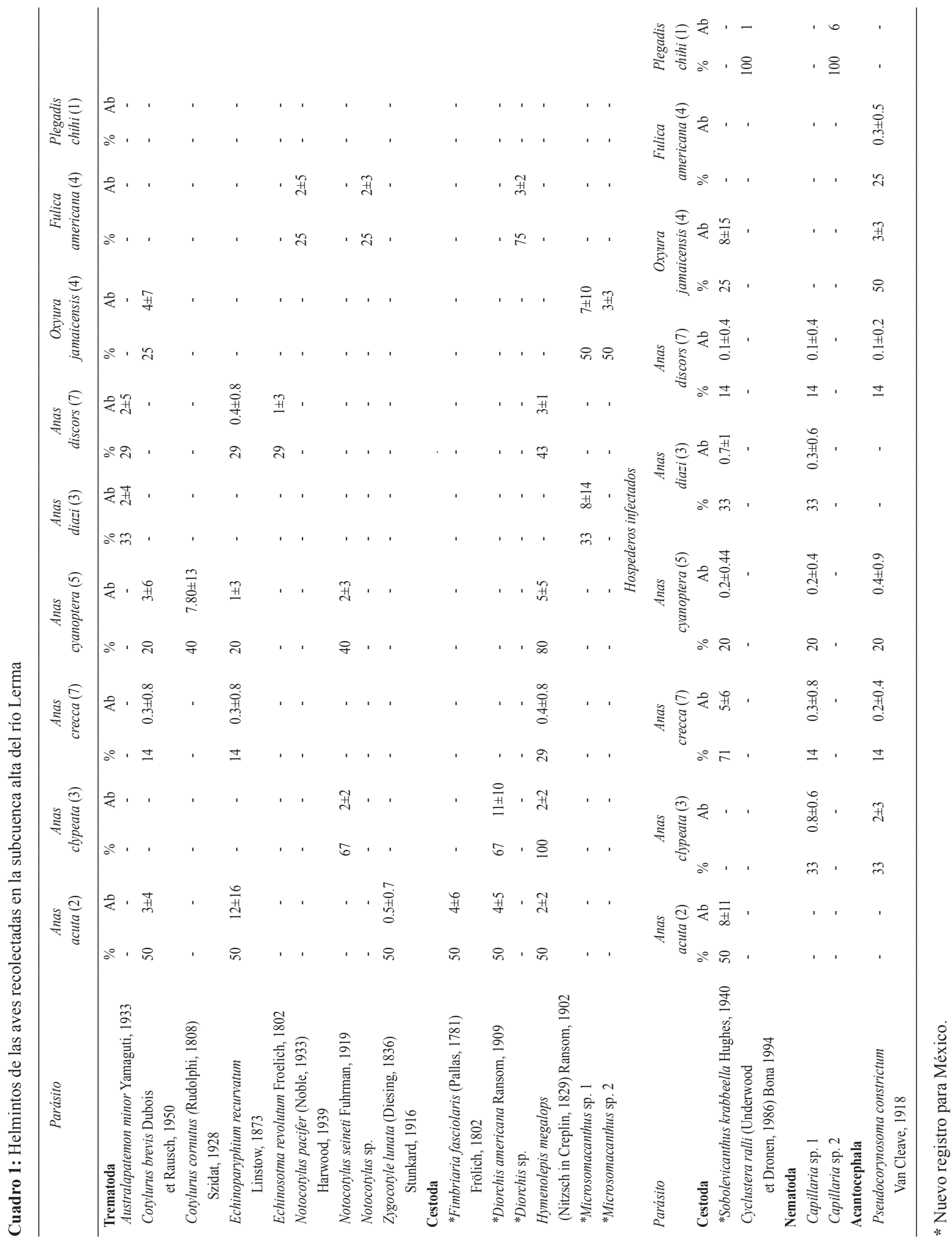


larvarias así como la obtención de helmintos inmaduros en los muestreos sugiere que éstos se adquirieron localmente a través del alimento en el área de estudio y al encontrar abundancias bajas, se dedujo que probablemente muchos sí se perdieron durante la ruta migratoria, pero de igual forma tanto la simpatría como la vagilidad de las aves migratorias y residentes permite ampliar la distribución geográfica y la recombinación genética de los helmintos. Otros factores que pueden influenciar la estructura de la comunidad de la helmintofauna gastrointestinal aviar, los cuales no fueron considerados en este estudio, son la eutroficación del ecosistema acuático y la existencia y tamaño poblacional de todos los hospederos que intervienen en los ciclos de vida de estos parásitos (Poulin, 2007).

\section{Agradecimientos}

A los señores Lucio Franco, Jorge Mercado, Ricardo Padilla, Alberto Guerrero y Gustavo Tovar, por la donación de las aves. A Sandra Hidalgo, Laura Patricia Becerril, Liliana Aranda y Joel Cruz, por su ayuda durante el examen helmintológico. Al Dr. Marcelo Silva Briano por las facilidades brindadas en el laboratorio de ecología de la Universidad Autónoma de Aguascalientes.

\section{Literatura citada}

Anderson, R., A. Chabaud y S. Willmott. 1986. Keys to the nematode parasites of vertebrates. Oxford University Press, New York. 480 p.

Barragán-Sáenz, F. A., P. Sánchez-Nava, O. Hernández.-Gallegos y G. Salgado-Maldonado. 2009. Larval stages of trematodes in gastropods from lake Chicnahuapan, State of Mexico, Mexico. Parasitology Research 105:1163-1167.

Broderson, D., A. G. Canaris y J. R. Bristol. 1977. Parasites of waterfowl from Southwest Texas: II. The shoveler, Anas clypeata. Journal of Wildlife Diseases 13:435-438.

Canaris, A. G., A. C. Mena y J. R. Bristol. 1981. Parasites of waterfowl from Southwest Texas: III. The Green-winged Teal, Anas crecca. Journal of Wildlife Diseases 17:57-64.

Canaris, A. G. y H. Lei-Ching. 1989. Levinseniella yucatanensis n. sp. (Digenea: Microphallidae) and other parasites from the Blue-winged Teal, Anas discors, from Yucatan, Mexico. Journal Parasitology 75:669-672.

Colón-Quezada, D. 2009. Composición de la dieta de otoño del pato mexicano (Anas diazi) en el vaso sur de las ciénegas del Lerma, Estado de México. Revista Mexicana de Biodiversidad 80:193-202.

Czaplinski, B. y C. Vaucher. 1994. Family Hymenolepididae Ariola, 1899. In Keys to the cestode parasites of vertebrates, L. F. Khalil, A. Jones and R. A. Bray (eds.) Commonwealth Agriculture Bureaux International, Wallingford. p. 595-663.
Digiani, M. C. 2000. Digeneans and cestodes parasiti in the withe-faced ibis Plegadis chihi (Aves: Threskiornithidae) from Argentina. Folia Parasitologica 47:195-204.

Farias, J. D. y A. G. Canaris. 1986. Gastrointestinal helminths of the mexican duck Anas platyrhynchos diazi ridgway, from north central Mexico and southwestern United States. Journal of Wildlife Diseases 22:51-54.

Fedynich, A. M. y D. B. Pence. 1994. Helminth community structure and pattern in a migratory host (Anas platyrhynchos). Canadian Journal of Zoology 72:496-505.

Fedynich, A. M., D. B. Pence y J. F. Bergan. 1996. Helminth community structure and pattern in sympatric populations of black-bellied and fulvous whistling-ducks. Canadian Journal of Zoology 74:2219-2225.

García-Prieto, L., M. García-Varela, B. Mendoza-Garfias y G. Pérez-Ponce de León. 2010. Checklist of the Acanthocephala in wildlife vertebrates of Mexico. Zootaxa 2419:1-50.

Gibson, I. D., A. Jones y R. A. Bray. 2002. Keys to the Trematoda, vol 1. CABI/ The Natural History Museum, London. 521 p.

Gladden, B. W. y A. G. Canaris. 2009. Helminth parasites of the Bufflehead duck, Bucephala albeola, wintering in the Chihuahua desert with a checklist of helminth parasites reported from this host. Journal Parasitology 95:129-136.

Haukos, D. A. y J. Neaville. 2003. Spatial and temporal changes in prevalence of a cloacal cestode in wintering waterfowl along y the Gulf Coast Texas. Journal of Wildlife Diseases 39:153-160

Howell, S. N. G. y S. Webb. 1995. A guide to the birds of Mexico and northern Central America. Oxford University Press, New York. 851 p.

Jones, A. 1994. Family Dilepididiae Railliet y Henry, 1909. In Keys to the cestode parasites of vertebrates, L. F. Khalil, A. Jones y R. A. Bray (eds.). Commonwealth Agriculture Bureaux International, Wallingford. p. 443-554.

Jones, A., R. A. Bray y D. I. Gibson. 2005. Keys to the Trematoda, vol 745. CABI/ The Natural History Museum, London. 521 p.

Krebs, C. J. 1999. Ecological methodology, segunda edición. Addison Wesley Longman, Reading, CA. 620 p.

Margolis, L., G. W. Esch, J. C. Holmes, A. M. Kuris y G. A. Schad. 1982. The use of ecological terms in parasitology (report of an ad hoc committee of the American Society of Parasitologists). Journal Parasitology 68:131-133.

McDonald, M. E. 1988. Key to Acanthocephala reported in waterfowl. US Department of the Interior Fish and Wildlife Service, Washington, D. C. 23 p.

Ortega-Olivares, M. P., A. O. Barrera-Guzmán, I. Haasova, G. Salgado-Maldonado, S. Guillén-Hernández y T. Scholz. 2008. Tapeworms (Cestoda: Gryporhynchidae) of fisheating birds (Ciconiformes) from Mexico: new host and geographical records. Comparative Parasitology 75:182-195.

Pérez-Ponce de León, G., L. García-Prieto y B. Mendoza-Garfias. 
2007. Trematode parasites (Platyhelminthes) of wildlife vertebrates in Mexico. Zootaxa 1534:1-247.

Poulin, R. 2007. Evolutionary Ecology of Parasites. Second edition. Princeton University Press, New Jersey. 332 p.

RAMSAR. 2010. The List of Wetlands of International Importance. Convención relativa a los humedales a los humedales de importancia internacional; http//www.ramsar. $\mathrm{org} / \mathrm{cda} /$ es/ramsar-pubs-annolist-annotated-ramsar-16517 / main/ramsar/1-30-168\%5E16517_4000_2_; última consulta 15.XL.2010.

Salgado-Maldonado, G. 1979. Procedimientos y técnicas generales empleados en los estudios helmintológicos. Departamento de Pesca, Dirección General de Acuicultura, SAGARPA, México, D.F. 53 p.

Sánchez-Nava, P. 2004. Helmintos parásitos de Girardinichthys multiradiatus (Pisces: Goodeidae) en la subcuenca alta del Lerma. Tesis Doctorado Instituto de Biología, UNAM, México, D. F. 135 p.
Sánchez-Nava, P., G. Salgado-Maldonado, E. Soto-Galera y B. Jaimes-Cruz. 2004. Helminth parasites of Girardinichthys multiradiatus (Pises: Goodeidae) in the upper Lerma River sub-basin, Mexico. Parasitology Research 93:396-402.

Scholz, T., R. Kutcha y G. Salgado-Maldonado. 2002. Cestodes of the family Dilepididae (Cestoda: Cyclophyllidea) from fish-eating birds in Mexico: a survey of species. Systematic Parasitology 52:171-182.

Shaw, M. G. y A. Kocan. 1980. Helminth fauna of waterfowl in Central Oklahoma. Journal of Wildlife Diseases 16:59-64.

Watson, J. J. y A.W. Pike. 1993. Variation in the morphology of adult Apatemon gracilis Rudolphi, 1819 (Digenea: Strigeidae) reared in different avian hosts. Systematic Parasitology 26:33-38.

Wilkinson, J. N., A. G. Canaris y D. Broderson. 1977. Parasites of waterfowl from southwest Texas: I. The Northern Cinnamon Teal, Anas cyanoptera septentrionalium. Journal of Wildlife Diseases 13:62-63. 